\title{
No dejen nunca de caminar mirando al horizonte
}

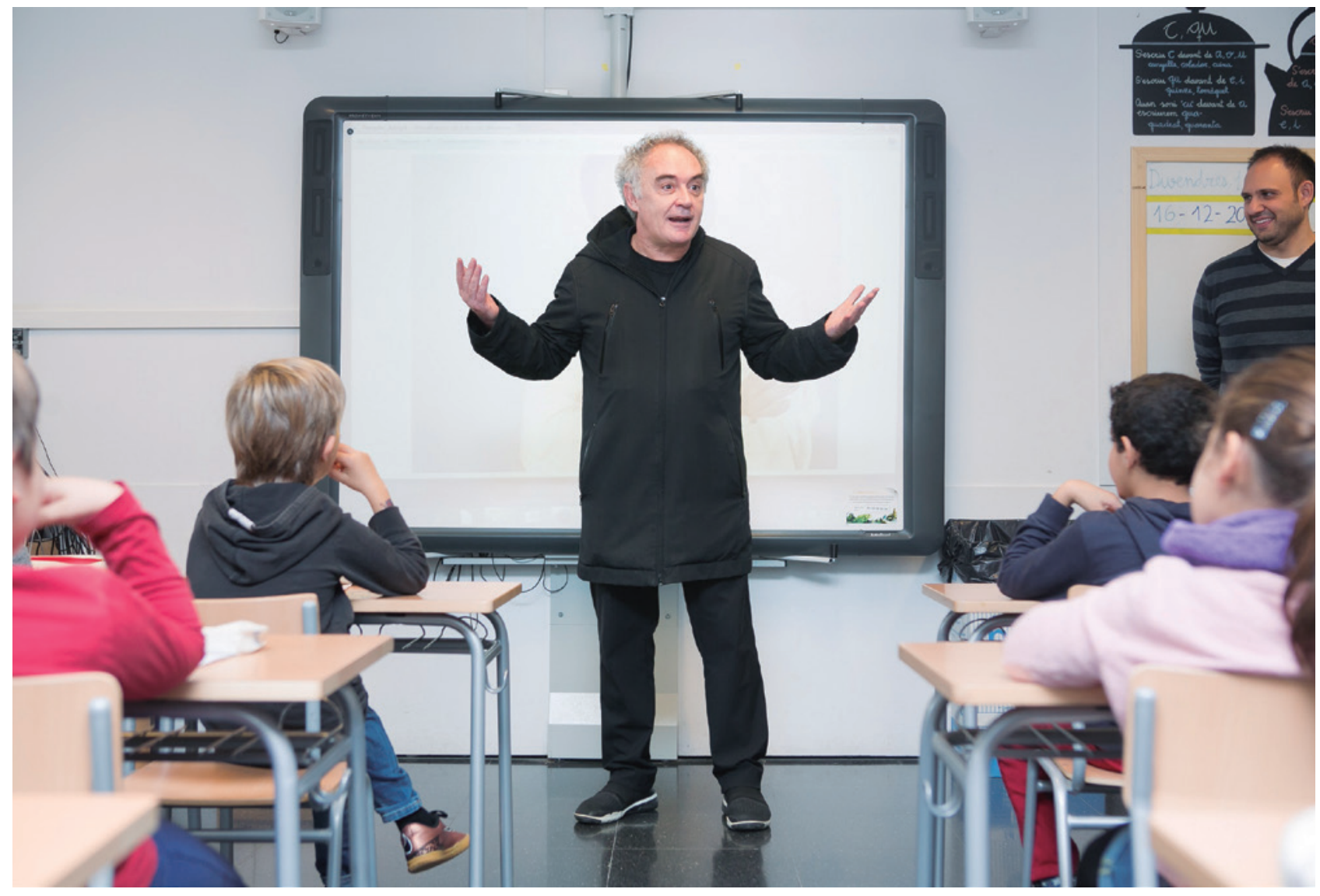

El proyecto "Escuelas Creativas" surge de la convicción de que cada realidad escolar es distinta. La clave ha sido entender que todas las escuelas tienen la capacidad interna de mejora

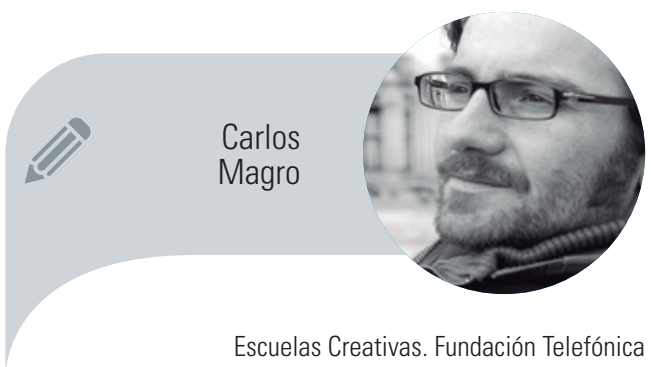
carlos.magromazo@gmail.com

y que cada comunidad educativa debe buscar

sus propias soluciones y fomentar y gestionar el cambio.

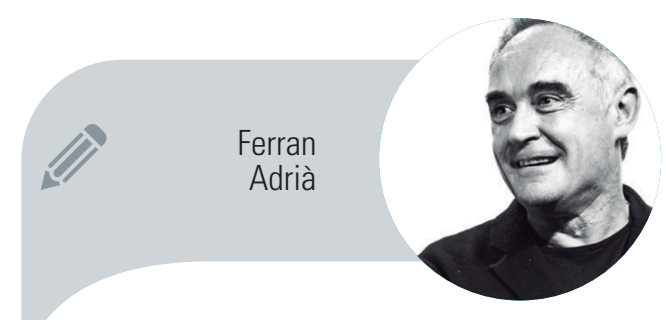


«La utopía está en el horizonte. Me acerco dos pasos, ella se aleja dos pasos. Camino diez pasos y el horizonte se desplaza diez pasos más allá. Por mucho que camine, nunca la alcanzaré. Entonces, ¿para qué sirve la utopía? Para eso: sirve para caminar» EduARdo Galeano

¿Qué tiene que ver la cocina con la educación? ¿Qué tienen en común un restaurante y un centro educativo? ¿Una cocina y un aula? ¿Se parecen en algo un equipo de cocineros y un claustro docente? ¿No estaremos simplificando demasiado la complejidad del acto educativo? ¿A qué nos referimos exactamente cuando hablamos de innovación educativa? ¿Todas las escuelas deben innovar? ¿Por qué nos ha entrado esa obsesión por la innovación? ¿No nos estaremos dejando llevar por la moda de la innovación? ¿No estaremos siendo, cuando menos, ingenuos al pensar que lo que funcionó en un contexto determinado y en un ámbito concreto puede ser útil en algo tan distinto como la educación? ¿Las escuelas, fomentan o eliminan la creatividad? ¿Se puede aprender a ser creativo? ¿Cuál es la relación entre creatividad, innovación, escuela y cocina? Y en el fondo, ¿por qué todo esto? ¿Para qué tantas preguntas? Al final, ¿qué buscamos realmente? ¿Por qué queremos escuelas creativas, profesores creativos, alumnos creativos? ¿Qué buscamos detrás de la idea de innovación?

Déjenme que arriesgue una primera respuesta, que a muchos les parecerá obvia: lo que buscamos es mejorar la educación de nuestros alumnos y, por tanto, pensamos, ayudar a que tengan una vida plena.

Hablamos tanto de cambio y perseguimos con tanta insistencia la innovación porque queremos lo mejor, en términos educativos, para nuestros hijos. Porque sabemos que solo las personas capaces de adaptarse a los cambios y a los nuevos aprendizajes podrán encarar, con alguna garantía, el futuro. Lo que está realmente detrás de nuestra creciente preocupación en torno a la educación, lo que subyace debajo de todas estas preguntas, lo que realmente nos preocupa colectiva e indi-

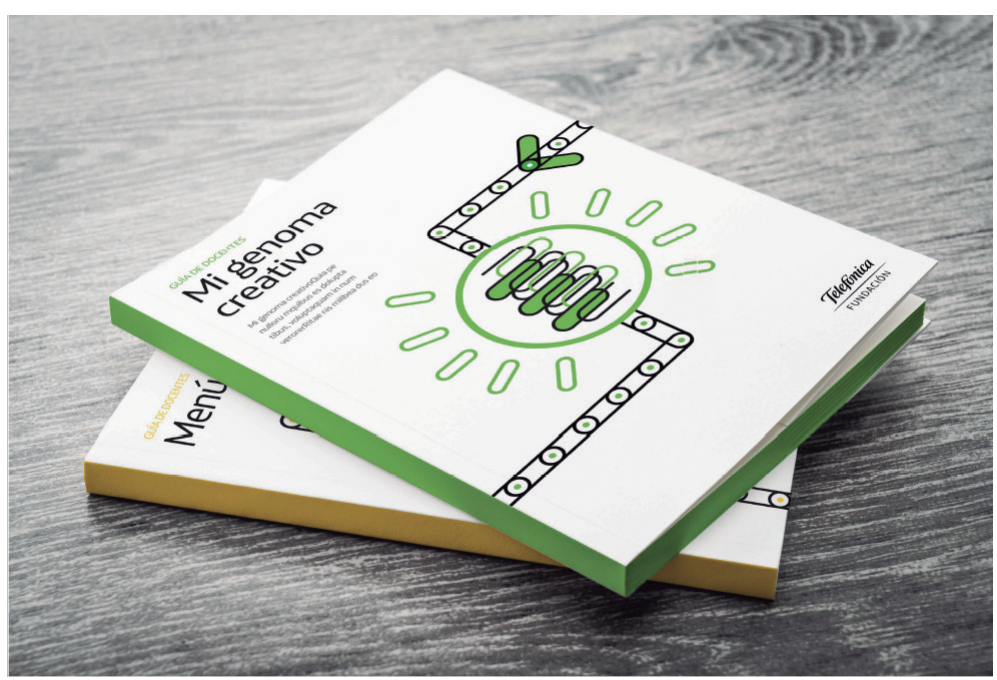

vidualmente, como maestros, como padres y como ciudadanos, lo que nos lleva a no estar nunca satisfechos, lo que nos impulsa a querer cambiar las cosas, a probar nuevas metodologías, a trabajar más allá de lo razonable es creer, como sostuvo el gran pedagogo brasileño Paulo Freire, que las cosas no son así, sino que están así y se pueden cambiar. Lo que nos mueve es creer que la educación es un factor de transformación, individual y colectivo. Lo que está detrás de las horas extras, los disgustos, las ilusiones y los esfuerzos de miles de maestras y maestros, madres y padres, a diario en todo el mundo es la convicción de que, como decía Jaume Carbonell parafraseando al poeta Gabriel Celaya, la educación al igual la poesía es un arma cargada de futuro.

Volvamos entonces sobre las preguntas. Las preguntas siempre son relevantes. Siempre son importantes y necesarias. Nunca sobran. Menos en educación. Son un dispositivo fundamental en el proceso de enseñanza/aprendizaje como bien defendió durante toda su vida el mismo Freire: "la educación de la respuesta no ayuda nada a la curiosidad indispensable para el proceso cognitivo. Al contrario, ella resalta la memorización mecánica de los contenidos. Sólo una educación de la pregunta agudiza, estimula y refuerza la curiosidad".

Y desde luego, en momentos como los actuales en los que tendemos a la respuesta rápida e irreflexiva, son más necesarias que nunca. Una pedagogía de la pregunta parece hoy un requisito básico para poder entender y vivir en una sociedad caracterizada por la incertidumbre, 


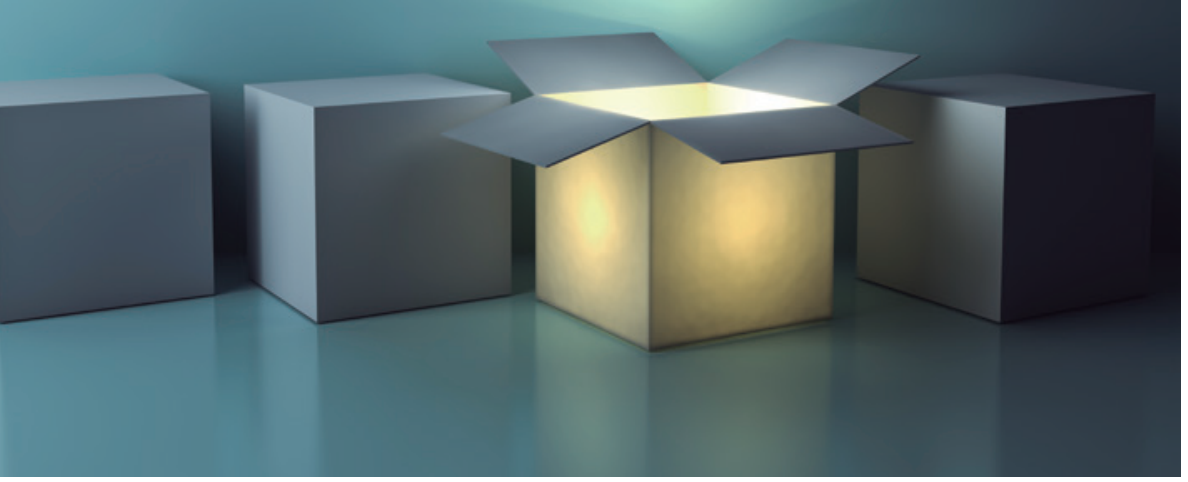

Educar es un acto creativo. Todas las escuelas son un lugar de creatividad porque no hay nada más creativo que un grupo de personas que, desde su diversidad, orientan sus esfuerzos a la larea de enseñar y de aprender juntos

la inseguridad, la flexibilidad, el relativismo y la ambigüedad. Las preguntas, si están bien hechas, facilitan el diálogo y nos abren el territorio de las posibilidades. Hoy, en educación, abundan las respuestas y faltan preguntas. Abundan las opiniones y escasea el debate. Hay un exceso tanto de visionarios como de inmovilistas. Parece que cualquiera es capaz de lanzar un diagnóstico sobre los males de la escuela y darnos su particular receta para la mejora.

Hacerse preguntas es clave en cualquier ámbito. Hacérselas hoy en educación es un asunto vital. Tenemos la oportunidad y la responsabilidad de trabajar por una educación mejor, por una educación transformadora. Es un buen momento de trabajar por la escuela que queremos.

La educación no es neutra. Todo acto educativo busca unos fines. Preguntar- nos por ese propósito, preguntarnos por los objetivos y por la finalidad última del proceso educativo, preguntarnos para qué educamos no es algo opcional, sino consustancial al hecho de educar. Y es una pregunta que debemos hacernos tanto a nivel individual como colectivo. A pesar de que formular preguntas y tratar de responderlas, más si es de manera colectiva, requiere de tiempo y disposición.

El problema con las preguntas difíciles es que tendemos a evitarlas o a simplificarlas. El problema de la educación es que son tantas las urgencias que hay que resolver, que apenas tenemos tiempo para dedicar tiempo a lo importante. El acierto del proyecto Escuelas Creativas fue entender y promover, desde el principio, entre los centros educativos participantes, la idea de que cualquier proceso de cambio debe partir de un proceso previo de reflexión de la comunidad educativa. El cambio y la mejora necesitan previamente de un análisis y un debate en torno a los objetivos del cambio, los fines de la educación, el tipo de aprendizaje que se quiere fomentar, el tipo de enseñanza que se requiere para desarrollar ese tipo de aprendizaje y, finalmente, sobre el modelo de liderazgo y organizativo que se necesita.

Necesitamos un aprendizaje más reflexivo y más alfabetización en la reflexión. La educación escolar necesita entornos donde el aprendizaje gire en torno a la reflexión y el pensamiento. Entornos donde no solo los alumnos aprendan a reflexionar sobre lo que hacen, sino donde también lo hagan los docentes. El cambio educativo que buscamos solo llegará a ser significativo si activa los procesos de acción-reflexión-acción en los sujetos que lo llevan a cabo (equipo directivo, docentes, alumnos, comunidad educativa) de forma participativa, cooperativa, negociada y deliberativa. Debemos analizar y reflexionar sobre lo que sucede a nivel de centro como dentro de las aulas.

El proyecto Escuelas Creativas surge de la convicción de que cada realidad escolar es distinta y que cada comunidad educativa es diferente. Que cada centro 


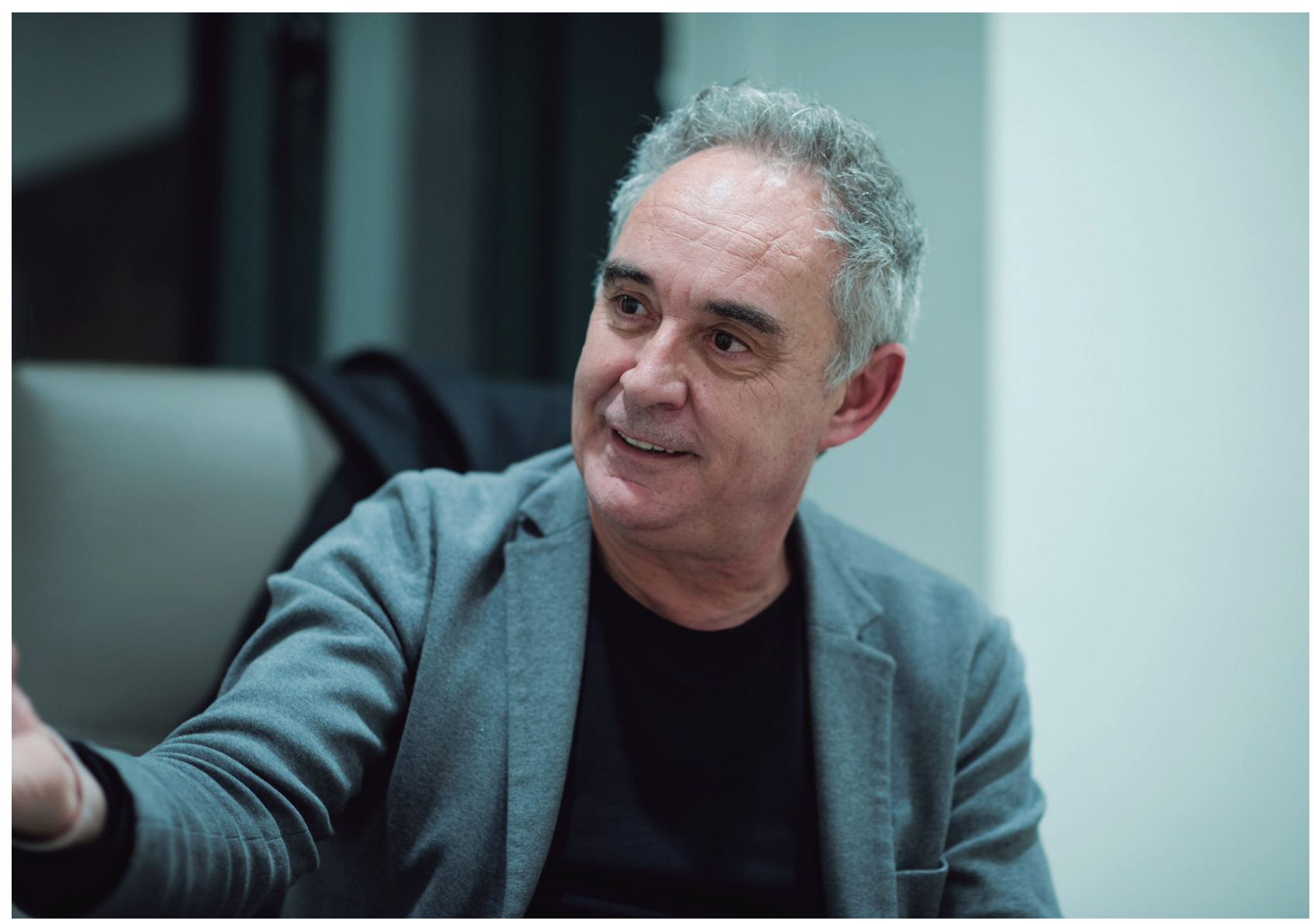

educativo es diferente al resto. Que aunque es mucho lo que comparten (un tronco de legislación común, unos currículos básicos comunes, una estructura y una organización escolar) también es mucho lo que los diferencia (legislación autonómica, titularidad del centro, nivel educativo, tamaño y líneas de escolaridad, estructura del claustro, docentes, alumnos, comunidad educativa, cultura escolar o manera de entender la educación).

El primer acierto del proyecto Escuelas Creativas ha sido entender que todas las escuelas tienen la capacidad interna de mejora y que nadie puede decir a otros lo que tienen que hacer. Que cada comunidad educativa debe buscar sus propias soluciones y fomentar y gestionar el cambio después. Que cualquier proceso de mejora escolar debe partir de un análisis previo de la situación del centro educativo (fortalezas y debilidades; datos, cultura escolar; contexto) que permita identificar las necesidades y áreas de mejora y establecer unas estrategias correctas. Que es necesario comprender para crear. Conocer para comprender y comprender para innovar. El conocimiento y la comprensión ganada nos permitirá visualizar y diseñar posteriormente una estrategia de innovación y creatividad para nuestro centro.

Con esa idea y con un claro propósito de ayudar y acompañar a centros, docentes y comunidades educativas en sus procesos de cambio, mejora, creatividad e innovación, el proyecto Escuelas Creativas ha desarrollado un amplio conjunto de materiales complementarios, cuyo núcleo central lo forman cinco guías que son el resultado del trabajo conjunto de un gran número de profesionales de la educación (docentes, directores, consultores educativos) que han trasladado al lenguaje y a las prácticas educativas las ideas que sobre innovación y cambio y sobre el proceso creativo hemos desarrollado en los últimos desde elBullifoundation.

Y aquí nos encontramos con un segundo acierto del proyecto. Involucrar desde el primer momento en el diseño y desarrollo del proyecto a docentes y profesionales de la educación, encargados de la adaptación y elaboración de los materiales de apoyo y también en el proceso de acompañamiento y asesoramiento a los centros. Materiales de apoyo pensados 


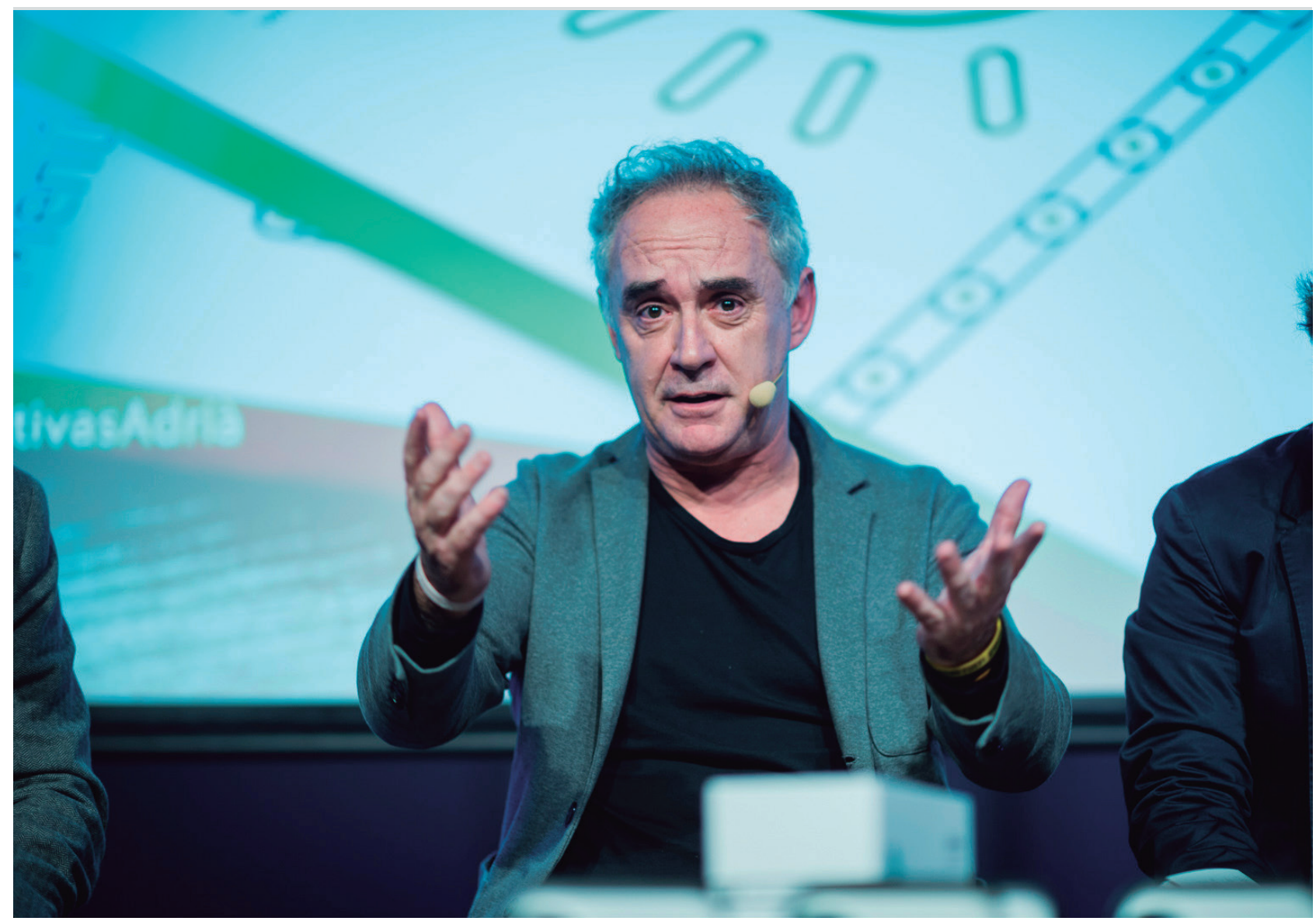

por educadores y profesionales de la educación, para educadores y otros profesionales de la educación.

Las guías tienen como objetivo ayudar y apoyar a los centros educativos y a los docentes en los procesos de cambio e innovación. Ayudarles a generar ecosistemas de aprendizaje creativo y que podemos agrupar en dos bloques según estén orientadas a nivel de centro (Sapiens Centro, Genoma de Centro) o a nivel de aula (EduSapiens, Mi Genoma Creativo, Menú de aprendizaje).

Las guías de Escuelas Creativas están llenas de recursos y herramientas para pensar, para innovar, para diseñar y trabajar la creatividad, para compartir experiencias y para mejorar. Herramientas para el cambio y la innovación, tanto del centro en su conjunto como de las prácticas de aula. Para trabajar con los alumnos, pero también para trabajar individualmente y con otros docentes. Materiales para ayudar a los centros a conocerse mejor, a analizar su contexto y sus procesos, a definir propuestas realistas de cambio y a llevarlas adelante. Materiales para que los profesionales de un centro se conozcan mejor individual y colectivamente. Materiales para desarrollar su creatividad y materiales también para desarrollar y trabajar la creatividad con los alumnos. Materiales para usar individualmente y colectivamente. Materiales para utilizar en el aula y fuera de la aula. Materiales abiertos, flexibles, modificables y adaptables.

Las escuelas no matan la creatividad. Todo lo contrario. Las escuelas son lugares de creatividad. No es que haya algunas escuelas y algunos docentes creativos. Es que todas las escuelas son creativas. Lo son porque todos lo somos. Porque la creatividad no es patrimonio de nadie, no pertenece a nadie y nadie tiene su exclusividad. Es un bien común y, como tal, es de todos y no es de nadie.

Las escuelas son creativas porque educar es un acto creativo o, como ha sostenido Gert Biesta, educar es un acto de creación de algo que no existía antes. O mejor, un acto de transformación. Todas las escuelas son un lugar de creatividad porque no hay nada más creativo que un grupo de personas que, desde su diversidad, orientan sus esfuerzos a la tarea de 
enseñar y de aprender juntos. No hay nada más creativo que la interacción que se produce a diario en un aula entre un maestro y sus alumnos. Esa interacción que llevó a Albert Camus a dedicar el discurso de aceptación del Premio Nobel de Literatura a su maestro y a escribirle días después una hermosa carta que todos quisiéramos recibir alguna vez. Por eso educamos.

La creatividad es, parafraseando a Jean Piaget, saber lo que hacer cuando no sabemos qué hacer. Así entendida, la creatividad, como la inteligencia es compuesta, expandible, práctica, intuitiva, distribuida, social. Y como la inteligencia, se puede desarrollar. Tenemos, de hecho, la responsabilidad de desarrollarla. Nacemos con enormes capacidades, pero esas capacidades se pueden ampliar todavía mucho más. La creatividad se aprende. Aprender, de hecho, nos hace más creativos y más inteligentes. Y en ese proceso, la escuela tiene mucho que decir.

Pero las escuelas también tienen ante sí el reto de la creatividad. El reto de desarrollar la creatividad de alumnos y docentes. El reto de convertirse en organizaciones más creativas, capaces de modificar y mejorar sus procesos y prácticas. El reto es convertirse en escuelas inteligentes, escuelas que aprenden, escuelas creativas.

Para que las escuelas se conviertan en escuelas que aprenden, estas deben ser no solo el lugar de aprendizaje para

\section{(口. DARA SABER MÂS}

Fundación TElefónicA. (2017). Escuelas Creativas. Un viaje hacia el cambio educativo. Fundación Telefónica.

https://www.fundaciontelefonica.com/ arte_cultura/publicaciones-listado/pagina-item-publicaciones/itempubli/612/

Fundación TelefónicA. (22 marzo 2018) Escuelas Creativas. Playlist. [vídeo]. Recuperado de https://www.youtube. com/playlist?list=PLMa9fq02Eqo89dsjn QUC9zMWn6cbbCEtN
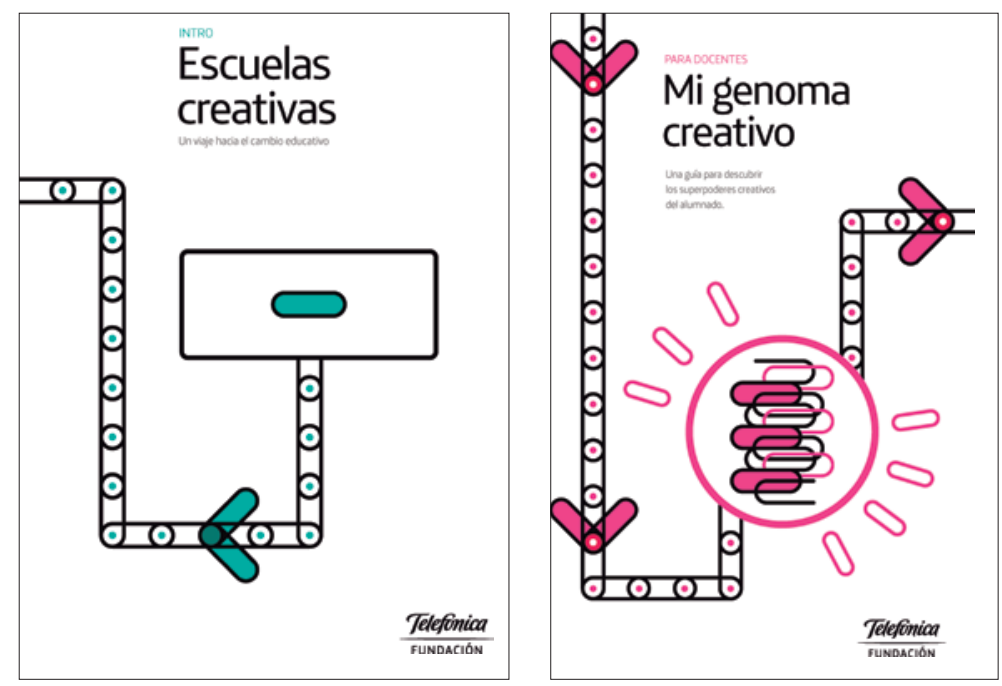

los alumnos, sino también un contexto estimulador del aprendizaje y crecimiento profesional de sus docentes.

La mejor manera que tienen las organizaciones de afrontar el futuro es creándolo. La mejor manera que tiene una escuela de preparar para el futuro es dotando a sus alumnos de las habilidades y la confianza en sí mismos para transformar sus entornos. No podemos predecir el futuro. Pero sí podemos soñarlo, imaginarlo y proyectarlo. Sí podemos construir una utopía que pase por imaginar visiones de futuro valientes, coherentes, inspiradoras y realistas.

En un mundo lleno de miradas catastrofistas sobre la educación, dice Axel Rivas, necesitamos más que nunca esperanzas practicables e inspiraciones alcanzables. El resultado de Escuelas Creativas es un buen conjunto de esperanzas practicables y, al menos, 17 inspiraciones alcanzables.

No dejen nunca de caminar mirando al horizonte •

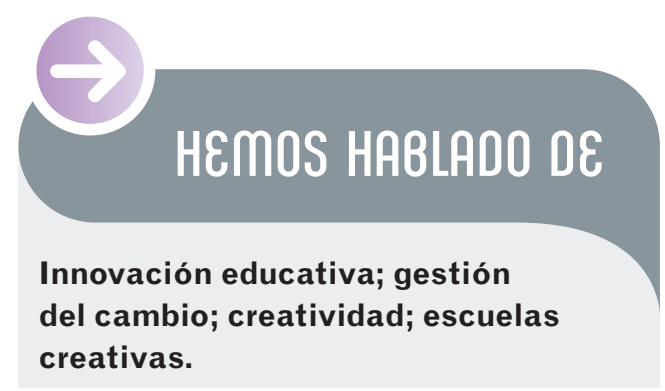

Este artículo fue solicitado por PADRES Y MAESTROS en diciembre de 2017, revisado y aceptado en marzo de 2018. 